\title{
Greedy Friensdhip Decompositions of Graphs*
}

\author{
Teresa Sousa \\ Departamento de Matem_atica, FCT-UNL and CMA-UNL, Quinta da Torre, Caparica, Portugal \\ E-mail:tmjs@fct.unl.pt \\ Received March 13, 2011; revised March 20, 2011; accepted April 2, 2011
}

\begin{abstract}
A graph that consists of $t$ cliques sharing a vertex $v$ is said to be a $t$-friendship graph with center $v$. A friendship graph is a graph that is $t$-friendship for some $t \geq 1$. We solve the problem of finding the best upper bound for the size of a greedy 2 -friendship decomposition and a greedy friendship decomposition of graphs of order $n$.
\end{abstract}

Keywords: Friendship Graph, Decompositions, Greedy Decompositions

\section{Introduction}

For notation and terminology not discussed here the reader is referred to [2]. All graphs considered here are finite and simple, i.e., they have no loops or multiple edges. Let $G$ be a simple graph with vertex set $V=V(G)$ and edge set $E=E(G)$. The degree of a vertex $V$ will be denoted by $\operatorname{deg}_{G^{v}}$ or briefly by $\operatorname{deg} v$ if it is clear which graph is being considered. A clique is a complete graph and the complete bipartite graph with parts of size $m$ and $n$ will be denoted by $K_{m, n}$. A graph that consists of $t$ cliques sharing a vertex $v$ is said to be a $t$-friendship graph ( $t$-fs graph) with center $v$. A friendship graph is a graph that is $t$-friendship for some $t \geq 1$. A ( $t$-)friendship decomposition of a graph $G$ is a set of pairwise edge disjoint ( $t$-)friendship subgraphs of $G$, such that every edge of $G$ appears in exactly one element of the decomposition. Let $\phi_{t}(G)$ be the minimum number of elements in a $t$-friendship decomposition of $G$. The main goal is to study the function

$$
\phi_{t}(n)=\max \left\{\phi_{t}(G) \mid v(G)=n\right\},
$$

which is the smallest number such that any graph $G$ of order $n$ admits a $t$-friendship decomposition with at most $\phi_{t}(n)$ elements.

Observe that a 1-friendship graph is just a clique. Erdös, Goodman and Pósa [3] proved that $\phi_{1}(n)=$ $\left\lfloor n^{2} / 4\right\rfloor$. Sousa [5] determined the exact value of the function $\phi_{t}(n)$ for $t=2$ and $t=3$, and for $t \geq 4$ its asymptotic value, as $n$ tends to infinity, was determined.

${ }^{*}$ This work was partially supported by Financiamento Base 2010 ISFL-1-297 from FCT/MCTES/PT.
More precisely, Sousa [5] proved that $\phi_{2}(n)$ equals $\left\lceil n^{2} / 8 \quad\right.$ if $n$ is even and $\left(n^{2}-1\right) / 8$ if $n$ is odd; and that $\phi_{3}(n)$ equals $\left\lceil n^{2} / 12\right\rceil$ if $n$ is even and $\left\lceil\left(n^{2}-1\right) / 12\right\rceil$ if $n$ is odd and not equal to 5. For $t \geq 4$ it was proved that $\phi_{t}(n)=n^{2} / 4 t+o\left(n^{2}\right)$.

In this paper our aim is to study the problem of looking not at an optimal friendship decomposition of graphs but at greedy friendship decompositions of graphs. The motivation for this work comes from the work or McGuinness [4] about clique decompositions of graphs, proving a conjecture of Winkler [1]. (Note that a clique is simple a 1-friendship graph.) McGuinness proved that if maximal cliques are removed one by one from any graph with $n$ vertices, then the graph will be empty after at most $n^{2} / 4$ steps. He also proves that this in fact best possible. The result is the following.

Theorem 1 [4] Any greedy clique decomposition of a graph with $n$ vertices has at most $\left|n^{2} / 4\right|$ cliques, and it has exactly $\left\lfloor n^{2} / 4\right\rfloor$ cliques if and only if the graph is $K_{\lfloor n / 2\rfloor,\lceil n / 2\rceil}$.

Note that the result of McGuinness is strong in the sense that for clique decompositions of graphs the optimal values are the same whether you consider optimal clique decompositions or greedy clique decompositions. With this work our goal is to see what happens if instead of 2-friendship decompositions of graphs one considers greedy 2-friendship decompositions of graphs. We will see later that the best values are not the same, in fact, they are quite far apart.

We start our work by properly defining the notion of a maximal ( $t$-)friendship subgraph of a graph. A ( $t$-)friendship subgraph of a graph $G$ is said to be maximal if it is not a proper subgraph of another ( $t$-)friendship subgraph 
of $G$ and its cliques can be ordered in such a way that each clique is maximal in the graph obtained after the edge set of the previous cliques have been removed. A greedy (t-)friendship decomposition of $G$ is formed by choosing a maximal ( $t$-)friendship graph in $G$, then choosing a maximal ( $t$-)friendship graph in the graph remaining after deleting the edges of the first ( $t$-)friendship graph and continuing removing maximal ( $t$-)friendship graphs until the graph is empty. We will always assume that the elements of the decomposition are chosen in a certain order.

For greedy 2-friendship decompositions we will prove that any greedy 2 -friendship decomposition of a graph of order $n$ has at most $\left.\mid n^{2} / 8+n / 4\right]$ elements. Furthermore, for $n \equiv 0,1,2(\bmod 4)$ a greedy 2 -friendship decomposition of a graph of order $n$ can have exactly $\left.\mid n^{2} / 8+n / 4\right\rfloor$ elements if and only if the graph is $K_{\lfloor n / 2\rfloor,\lceil n / 2\rceil}$. For $n \equiv 3(\bmod 4)$ a greedy 2 -friendship decomposition of $K_{\lfloor n / 2\rfloor,\lceil n / 2\rceil}$ can have exactly $\left\lfloor n^{2} / 8+n / 4\right\rfloor 2$-friendship graphs, however, in this case we can also have equality for non-bipartite graphs. We conclude by showing that any greedy friendship decomposition of a graph of order $n$ has at most $n-2$ elements and we also prove that this upper bound is, in fact, best possible.

\section{Greedy Friendship Decompositions}

We start this section with the following result about greedy 2-friendship decompositions of graphs and conclude with a result concerning greedy friendship decompositions of graphs.

Theorem 1 Any greedy 2-friendship decomposition of a graph of order $n$ has at most $\left\lfloor n^{2} / 8+n / 4\right\rfloor 2$-friendship graphs.

Proof. Let $G$ be a graph with $n$ vertices, where $n \geq 2$, and $\mathcal{F}$ a greedy 2-fs decomposition of $G$. Using the same order in which the elements of $\mathcal{F}$ were chosen we construct a greedy clique decomposition of $G, \mathcal{C}$, as follows:

Let $F \in \mathcal{F}$ and let $C_{1}, C_{2}$ be its cliques. Assume without loss of generality that $C_{1}$ is maximal, then we first add $C_{1}$ to $\mathcal{C}$ and then add $C_{2}$.

Clearly $\mathcal{C}$ is a greedy clique decomposition of $G$. Let $\mathcal{F}_{1}$ be the set of all 2-fs graphs in $\mathcal{F}$ that have exactly 1 clique and $\mathcal{F}_{2}$ the set of all 2-fs graphs in $\mathcal{F}$ that have exactly 2 cliques. Then, $|\mathcal{C}|=\left|\mathcal{F}_{1}\right|+2\left|\mathcal{F}_{2}\right|$. Observe that $\left|\mathcal{F}_{1}\right| \leq\lfloor n / 2\rfloor$. Therefore,

$$
|\mathcal{F}|=\left|\mathcal{F}_{1}\right|+\left|\mathcal{F}_{2}\right|=\left|\mathcal{F}_{1}\right|+\frac{|\mathcal{C}|-\left|\mathcal{F}_{1}\right|}{2} \leq \frac{1}{2}\left\lfloor\frac{n^{2}}{4}\right\rfloor+\frac{1}{2}\left\lfloor\frac{n}{2}\right\rfloor
$$

(by Theorem 1.1)

Easy calculations show that $\left\lfloor 1 / 2\left\lfloor n^{2} / 4\right\rfloor+1 / 2\lfloor n / 2\rfloor\right\rfloor$ $=\left|n^{2} / 8+n / 4\right|$ and the result follows.

Theorem 2 Let $n \equiv 0,1,2(\bmod 4)$ and let $G$ be $a$ graph of order $n$. Then $G$ admits a greedy 2-friendship decomposition with exactly $\left\lfloor n^{2} / 8+n / 4\right\rfloor$ elements if and only if $G=K_{\lfloor n / 2\rfloor,\lceil n / 2\rceil}$.

Proof. Let $\mathcal{F}$ be a greedy 2-friendship decomposition of the graph $G$ with exactly $\left\lfloor n^{2} / 8+n / 4\right\rfloor$ elements. Let $\mathcal{C}$ and $\mathcal{F}_{1}$ be as in the proof of Theorem 2.1. Suppose that $|\mathcal{C}| \leq\left|n^{2} / 4\right|-1$. From (2.1) we obtain

$$
\begin{aligned}
\left\lfloor n^{2} / 8+n / 4\right\rfloor= & \left\lfloor 1 / 2|\mathcal{C}|+1 / 2\left|\mathcal{F}_{1}\right|\right\rfloor \\
& \leq\left\lfloor 1 / 2\left\lfloor n^{2} / 4\right\rfloor-1 / 2+1 / 2\lfloor n / 2\rfloor\right\rfloor
\end{aligned}
$$

which is a contradiction for $n \equiv 0,1,2(\bmod 4)$. Therefore $|\mathcal{C}|=\left\lfloor n^{2} / 4\right\rfloor$ and Theorem 1 implies that $G=K_{\lfloor n / 2\rfloor,\lceil n / 2\rceil}$.

Now assume that $G=K_{\lfloor n / 2\rfloor,\lceil n / 2\rceil}$. It suffices to find a 2-fs decomposition of $G$, say $\mathcal{F}$, with exactly $n^{2} / 8+n / 4$ elements. Let $A, B$ be a partition of $G$ with $A=\left\{a_{i}|i=1, \cdots| A \mid,\right\} \quad$ and $\quad B=\left\{b_{i}|i=1, \cdots| B \mid,\right\} \quad$ and assume, without loss of generality, that $|A| \leq|B|$. We consider three different cases.

a) Assume that $n=4 k$ and construct $\mathcal{F}$ as follows:

i) For $i=1, \cdots, 2 k-2$, pair all the edges incident with $a_{i}$ except the edges $\left\{a_{i}, b_{2 k}\right\}$ and $\left\{a_{i}, b_{i}\right\}$, and for $i=2 k-1,2 k$, pair all the edges incident with $a_{i}$ except the edges $\left\{a_{i}, b_{1}\right\}$ and $\left\{a_{i}, b_{i}\right\}$. In total $\mathcal{F}$ will get $2 k(k-1)$ elements.

ii) Observe that $\operatorname{deg} b_{2 k}=2 k-1$, so we pair all the edges incident with $b_{2 k}$ except the edge $\left\{a_{2 k}, b_{2 k}\right\}$ to get $k-12$-fs graphs and we consider the 2-fs graph with edges $\left\{b_{1}, a_{2 k-1}\right\}$ and $\left\{b_{1}, a_{2 k}\right\}$. In total $\mathcal{F}$ will increase by $k$ elements.

iii) At this step the $2 k$ edges left form a perfect matching and we add them all to $\mathcal{F}$.

Thus,

$$
|\mathcal{F}|=2 k(k-1)+k+2 k=2 k^{2}+k=\left\lfloor n^{2} / 8+n / 4\right\rfloor
$$

b) Assume that $n=4 k+1$ and construct $\mathcal{F}$ as follows:

i) For $i=1, \cdots, 2 k$, pair all the edges incident with $a_{i}$ except edges $\left\{a_{i}, b_{i}\right\}$. In total $\mathcal{F}$ will get $2 k^{2}$ elements.

ii) At this step the $2 k$ edges left form a matching and we add them all to $\mathcal{F}$.

Therefore, $|\mathcal{F}|=2 k^{2}+2 k=\left\lfloor n^{2} / 8+n / 4\right\rfloor$.

c) Assume that $n=4 k+2$ and construct $\mathcal{F}$ as follows:

i) For $i=1, \cdots, 2 k+1$, pair all the edges incident with $a_{i}$ except edges $\left\{a_{i}, b_{i}\right\}$. In total $\mathcal{F}$ will get $(2 k+1) k$ elements.

ii) For $i=1, \cdots, 2 k+1$, pair all the edges incident with 
$a_{i}$ except edges $\left\{a_{i}, b_{i}\right\}$. In total $\mathcal{F}$ will get $(2 k+1) k$ elements.

Therefore,

$$
\begin{aligned}
|\mathcal{F}| & =(2 k+1) k+2 k+1 \\
& =2 k^{2}+3 k+1=\left\lfloor n^{2} / 8+n / 4\right\rfloor
\end{aligned}
$$

Remark: The following example shows that for $n \equiv 3(\bmod 4) \quad K_{\lfloor n / 2\rfloor,\lceil n / 2\rceil}$ is not the only extremal graph for greedy 2-friendship decompositions.

Example: Let $A=\left\{a_{1}, \cdots, a_{2 k+1}\right\}$ and $B=\left\{b_{1}, \cdots, b_{2 k+2}\right\}$ and let $G$ be the complete bipartite graph with parts $A$ and $B$ plus the edge $\left\{b_{2 k+1}, b_{2 k+2}\right\}$. Construct $\mathcal{F}$ as follows:

i) For $i=1, \cdots, 2 k+1$, pair all the edges incident with $b_{i}$ except edges $\left\{a_{i}, b_{i}\right\}$ and the edge $\left\{b_{2 k+1}, b_{2 k+2}\right\}$. In total $\mathcal{F}$ will get $(2 k+1) k$ elements.

ii) Pair all the edges incident with $b_{2 k+2}$ except the edges $\left\{a_{2 k+1}, b_{2 k+2}\right\}$ and $\left\{b_{2 k+1}, b_{2 k+2}\right\}$, then $\mathcal{F}$ will increase by $k$ elements.

iii) Finally, we add to $\mathcal{F}$ the single edges $\left\{a_{i}, b_{i}\right\}$, for $i=1, \cdots, 2 k$ and the 2-fs graph with edges $\left\{a_{2 k+1}, b_{2 k+1}\right\},\left\{b_{2 k+1}, b_{2 k+2}\right\}$ and $\left\{a_{2 k+1}, b_{2 k+2}\right\}$, thus we add $2 k+1$ elements to $\mathcal{F}$.

Therefore,

$$
|\mathcal{F}|=(2 k+2) k+2 k+1=2 k^{2}+4 k+1=\left\lfloor n^{2} / 8+n / 4\right\rfloor
$$

and the graph $G$ is not bipartite.

We conclude this section with the following trivial theorem on the size of a greedy friendship decomposition of a graph.
Theorem 3 Any greedy friendship decomposition of a graph of order $n$, where $n \geq 3$, has at most $n-2$ elements. Furthermore, this bound is sharp for the bipartite graph $K_{2, n-2}$.

Proof. Let $G$ be a graph with $n \geq 3$ vertices. We will proceed by induction on $n$. If $n=3$ then the edges of $G$ form either a triangle or path of length 1 or 2, thus any friendship decomposition of $G$ will have exactly one element. Suppose the result holds for graphs with less than $n$ vertices. Let $\mathcal{F}$ be a greedy friendship decomposition $G$, let $F$ be the first friendship graph picked for $\mathcal{F}$ and let $v$ be its center. Consider the graph $H$ with vertex set $V(G)-\{v\}$ and $E(H)=E(G)-E(F)$. Clearly $\mathcal{F}-\{F\}$ is a greedy friendship decomposition of $H$, so by induction $|\mathcal{F}-\{F\}| \leq n-3$, hence the result.

\section{References}

[1] J. Bang-Jensen and B. Toft. Unsolved problems presented at the Julius Petersen Graph Theory Conference. Discrete Math., 101(1-3): 351-360, 1992. doi:10.1016/0012-365X(92)90616-N

[2] R. Diestel. Graph Theory. Springer-Verlag, 2nd edition, 2000.

[3] P. Erdös, A. W. Goodman, and L. Pósa, The representation of a graph by set intersections, Canad. J. Math. 18: 106-112, 1966. doi:10.4153/CJM-1966-014-3

[4] S. McGuinness. The greedy clique decomposition of a graph. J. Graph Theory, 18: 427-430, 1994. doi:10.1002/jgt.3190180412

[5] T. Sousa. Friendship decompositions of graphs. Discrete Math., 308(15): 3352-3360, 2008. doi:10.1016/j.disc.2007.06.042 